\title{
DE
}

DE GRUYTER

OPEN

BULGARIAN ACADEMY OF SCIENCES

CYBERNETICS AND INFORMATION TECHNOLOGIES • Volume 14, No 3

Sofia $\bullet 2014$

Print ISSN: 1311-9702; Online ISSN: 1314-4081

DOI: $10.2478 /$ cait-2014-0029

\section{Stability and Fractal Patterns of Complex Logistic Map}

\section{Bhagwati Prasad, Kuldip Katiyar}

Department of Mathematics, Jaypee Institute of Information Technology, A-10, Sector-62, Noida, UP-201307 INDIA

Emails: b_prasad10@yahoo.com, kuldipkatiyar.jiitn@gmail.com

Abstract: The intent of this paper is to study the fractal patterns of one dimensional complex logistic map by finding the optimum values of the control parameter using Ishikawa iterative scheme. The logistic map is shown to have bounded and stable behaviour for larger values of the control parameter. This is well depicted via time series analysis and interesting fractal patterns as well are presented.

Keywords: Complex logistic map, Ishikawa iteration, fractals, Julia set.

\section{Introduction}

The name "logistic growth model" is essentially due to Verhulst [20] which he used for the studies on population dynamics. He introduced logistic equation for demographic modelling by extending the Malthus equation of the continuous growth of a population with a view to obtain a stable stationary finite state (see [4]). It took more than hundred years to recognize his founding contributions towards the population dynamics and non-linear sciences. This work received wide attention due to the great implications of the simple looking equation in Chaos theory. In 1963, Edward Lorenz introduced an equivalent version of the logistic model for his famous weather forecast model [13]. R. M ay [15, 16], in 1976, recognized the importance of the logistic model and observed that the continuous time model may not be suitable to reflect the realities in most of the cases and constructed a discrete 
version of this model. Thereafter, Feigenbaum [8] and the work of others approved this model as the paradigm for the period doubling route to chaos [13]. The importance of this model is due to its peculiar behaviour for changing values of the parameter. It exhibits the fixed points, bifurcations and chaos for the successive values of the growth parameter. This provides the basis of the modern chaos theory and represents the simplest cases of chaotic system [3]. The extreme sensitivity to the initial conditions has made it to be ideal for various applications. Many variants and generalizations of this model have been used to study various physical problems. Dettmer [6] pointed out that the most obvious reason for knowing about chaos is to organize and possibly avoid it because the regularity and stability disappears once the system becomes chaotic. Generally feedback linearisation, variable structure controller, fuzzy method and neural networks etc. are among the various techniques used for controlling the chaos in the literature. Due to the advancement of modern computational tools and proliferation of digital computers, new vistas have been opened for the study and analysis of these hyper sensitive maps and the literature is flourished with the papers signifying their importance in chaos, fractals, cryptography, optimization, discrete dynamics, population dynamics etc. (see for instance, $[5,6,11,17,18,19,21,22,26,28]$ and several references thereof). Kint et al. [13] explored the graphical potential of this map and generated fractal figures comparable to the well known Mandelbrot fractals. They named these attracting fractal figures as Verhulst fractals. Recently Rani and A garwal [27] studied the comparative behaviour of the complex logistic maps with Picard orbit, Norland orbit and Mann orbit and found interesting results. Our aim is to study the stability of the logistic map for Ishikawa iterates and visualize the fractal patterns of such map for varying values of the parameters. We use the Matlab tools for all our computational and graphical requirement.

\section{Preliminaries}

Let $(Y, d)$ be a metric space and $f$ be a transformation on $Y$. Then $f$ may be called a dynamical system in the sense of B arnsley [1] and it is denoted by $\{Y, f\}$. The orbit of a point $x$ in $Y$ is defined as a sequence of iterates of $f$ in the form of $\left\{f^{n}(x)\right.$ : $n=0,1,2, \ldots\}$. Different iterative schemes have been used in the literature to obtain the orbits of such a dynamical system. The function iteration also known as Picard iteration is popularly used in the literature and its orbit (Picard Orbit - PO) is represented as

$$
\mathrm{PO}\left(f, x_{0}\right):=\left\{x_{n}: x_{n}=f\left(x_{n-1}\right), n=1,2, \ldots\right\} .
$$

This iteration requires one number as input to return a new number as output and popularly called as one step feedback machine. A two step feedback scheme requiring two numbers as input to return a new number as output is used by R a $\mathrm{i}$ and A g a r w a 1 [26] for the study of the chaotic behaviours of the logistic map. The $n$-th, $n=1,2,3, \ldots$, iterate of this is given as $x_{n}=\alpha_{n} f\left(x_{n-1}\right)+\left(1-\alpha_{n}\right) x_{n-1}$, where $0<\alpha_{n} \leq 1$ and the sequence $\left\{\alpha_{n}\right\}$ is converging away from 0 . The orbit generated 
using this scheme is called Mann Orbit (MO) or superior orbit (see [14]) and it may be represented in the following manner:

$$
\operatorname{MO}\left(f, x_{0}, \alpha_{n}\right):=\left\{x_{n}: x_{n}=\alpha_{n} f\left(x_{n-1}\right)+\left(1-\alpha_{n}\right) x_{n-1}, n=1,2,3, \ldots\right\} .
$$
[10].

Now we define a three step feedback scheme essentially due to I shikaw a

Definition 2.1. Let $Y$ be a non-empty set and $f: Y \rightarrow Y$. For a point $x_{0}$ in $Y$, construct a sequence $\left\{x_{n}\right\}$ in the following manner:

$$
\begin{aligned}
y_{n-1} & =\beta_{n} f\left(x_{n-1}\right)+\left(1-\beta_{n}\right) x_{n-1}, \\
x_{n} & =\alpha_{n} f\left(y_{n-1}\right)+\left(1-\alpha_{n}\right) x_{n-1},
\end{aligned}
$$

for $n=1,2,3, \ldots$, where $0<\alpha_{n} \leq 1$ and $0 \leq \beta_{n} \leq 1$ and $\left\{\alpha_{n}\right\}$ is convergent away from 0 . Then sequence $\left\{x_{n}\right\}$ constructed above will be called the Ishikawa iteration of a point $x_{0}$ and it is denoted by $\operatorname{IO}\left(f, x_{0}, \alpha_{n}, \beta_{n}\right)$. We shall study the Ishikawa Orbit (IO) for $\alpha_{n}=\alpha$ and $\beta_{n}=\beta$.

It is remarked that (3) becomes (2) when we put $\beta_{n}=0$ in it and (2) with $\alpha_{n}=1$ is the Picard iteration (1).

This scheme is widely studied by Prasad and Katiyar [23, 25] and interesting fractal patterns are generated in [24] using it.

Definition 2.2 [2]. Let $Y$ be a non-empty set and $f: Y \rightarrow Y$. A point $p \in Y$ is called a periodic point of $f$ of period $n \geq 1, n \in N$ (the set of natural numbers), iff $f^{n}(p)=p$ and $f^{k}(p) \neq p$ for all $k=1,2, \ldots, n-1$, where $f^{k}(p)$ is the $k$-th iterate of point $p$ under $f$. A periodic point of $f$ of period 1 is simply a fixed point of $f$.

Definition 2.3 [9]. Let $f$ be a function and $p$ be a periodic point of $f$ with prime period $k$. Then $x$ is forward asymptotic to $p$ if the sequence $x, f^{k}(x), f^{2 k}(x), f^{3 k}(x), \ldots$ converges to $p$. In other words, $\lim _{n \rightarrow \infty} f^{n k}(x)=p$. The stable set of $p$, denoted by $W^{s}(p)$, consists of all points which are forward asymptotic to $p$. If the sequence $|x|$, $\left|f^{k}(x)\right|,\left|f^{2 k}(x)\right|,\left|f^{\beta k}(x)\right|, \ldots$ grows without bound, then $x$ is forward asymptotic to $\infty$. The stable set of $\infty$, denoted by $W^{s}(\infty)$, consists of all points which are forward asymptotic to $\infty$.

The following definition is motivated by Rani and Agarwal [26].

Definition 2.4 [26]. Let $S \subset R$ (the set of real numbers), $f: S \rightarrow S$ and $p$ is a periodic point of $f$ with prime period $k$. For a point $x_{0} \in S$ and $p \in[0,1]$, construct a sequence $\left\{x_{n}: n=1,2, \ldots\right\}$ such that

$$
\begin{gathered}
y_{0}=(1-\beta) x_{0}+\beta f\left(x_{0}\right), \\
x_{k}=(1-\alpha) x_{0}+\alpha f\left(y_{0}\right), \\
y_{k}=(1-\beta) x_{k}+\beta f\left(x_{k}\right), \ldots \\
x_{2 k}=(1-\alpha) x_{k}+\alpha f\left(y_{k}\right), \\
y_{(n-1) k}=(1-\beta) x_{(n-1) k}+\beta f\left(x_{(n-1) k}\right), \ldots
\end{gathered}
$$




$$
x_{n k}=(1-\alpha) x_{(n-1) k}+\alpha f\left(y_{(n-1) k}\right) .
$$

Then $x_{0}$ is called Ishikawa forward asymptotic to $p$ and sequence $\left\{x_{n k}\right\}$ converges to $p$.

Definition 2.5 [1]. Let $C$ be the complex plane, $\widehat{C}=C \cup\{\infty\}$ and $f: \widehat{C} \rightarrow \widehat{C}$ denote a polynomial of degree greater than 1 . Let $F_{f}$ denote the set of points in $\hat{C}$ whose orbits do not converge to the point at infinity. That is,

$$
F_{f}=\left\{z \in \widehat{C}:\left\{\left|f^{n}(z)\right|\right\}_{n=0}^{\infty} \text { is bounded }\right\} .
$$

This set is called the filled Julia set associated with the polynomial $f$. The boundary of $F_{f}$ is called the Julia set of the polynomial $f$ and is denoted by $J_{f}$.

\section{Discussions and results}

Verhulst postulated that the growth rate at any time should be proportional to the fraction of the environment that is not yet used up by the population at that time and thus formulated the following model:

$$
p_{n+1}=p_{n}+a p_{n}\left(1-p_{n}\right),
$$

here $p_{n}$ measures the relative population count at time $n$ and $a$, the growth rate at time $n$ measures the increase of the population in one time step relative to the size of the population at that time [21]. Verhlust's model was further expressed by R. May in the following manner:

$$
X_{n+1}=r X_{n}\left(1-X_{n}\right),
$$

where $X_{n}$ (a real number between 0 and 1) represents population density at time $n=1,2,3, \ldots$ and $r$ (a non-negative real number) is used for the combined rate for reproduction and starvation [9].

The quadratic transformations of the type $z \rightarrow z^{2}+c$, where $z$ and $c$ both are from complex plane $C$, are widely studied by $[1,7,12,22]$ and many others in the literature. The interest is to know the behaviour of the structure of the orbit of the iterates of $z$ when $z$ and $c$ vary. For $n=0,1,2, \ldots$ the iteration scheme of such map is

$$
z_{n+1}=z_{n}^{2}+c .
$$

Peitgen et al. [21] have established the equivalence of the maps given above. Following them, one can easily see that equations (4) and (6) are identical for $c=\left(1-a^{2}\right) / 4, \quad z_{n}=(1+a) / 2-a p_{n}$, whereas (4) and (5) are identical for $X_{n}=a p_{n} /(1+a), r=1+a$. On simplifying, we find that (5) and (6) become identical for $c=r(2-r) / 4, z_{n}=r / 2-r X_{n}$. This functional equivalence is useful to generate the fractal patterns for the quadratic map given by (6) after obtaining $c$ in terms of $r$. 
First we study the behaviour of the map given by (5) when $X$ and $r$ are complex numbers. Let $X_{n}=X_{x_{n}}+i X_{y_{n}}, r=r_{x}+i r_{y}$. Now we compute the values of $X_{x_{n}}$ and $X_{y_{n}}$ at different iteration levels using the iterative scheme (3) in the following manner

$$
\begin{aligned}
& X_{x_{n+1}}=\alpha\left\{r_{x}\left(X_{x_{n}}^{\prime}-X_{x_{n}}^{\prime 2}+X_{y_{n}}^{\prime 2}\right)-r_{y}\left(X_{y_{n}}^{\prime}-2 X_{x_{n}}^{\prime} X_{y_{n}}^{\prime}\right)\right\}+(1-\alpha) X_{x_{n}}, \\
& X_{y_{n+1}}=\alpha\left\{r_{x}\left(X_{y_{n}}^{\prime}-2 X_{x_{n}}^{\prime} X_{y_{n}}^{\prime}{ }^{2}+r_{y}\left(X_{x_{n}}^{\prime}-X_{x_{n}}^{\prime 2}+X_{y_{n}}^{\prime 2}\right)\right\}+(1-\alpha) X_{y_{n}},\right.
\end{aligned}
$$

where

$$
\begin{aligned}
& X_{x_{n}}^{\prime}=\beta\left\{r_{x}\left(X_{x_{n}}-X_{x_{n}}{ }^{2}+X_{y_{n}}{ }^{2}\right)-r_{y}\left(X_{y_{n}}-2 X_{x_{n}} X_{y_{n}}\right)\right\}+(1-\beta) X_{x_{n}}, \\
& X_{y_{n}}^{\prime}=\beta\left\{r_{x}\left(X_{y_{n}}-2 X_{x_{n}} X_{y_{n}}{ }^{2}+r_{y}\left(X_{x_{n}}-X_{x_{n}}{ }^{2}+X_{y_{n}}{ }^{2}\right)\right\}+(1-\beta) X_{y_{n}},\right.
\end{aligned}
$$

and find the optimum value of $|r|$ for various choices of parameters $\alpha, \beta$. We consider $X_{0}=X_{x_{0}}+i X_{y_{0}}=0.01+0.01 i$ as the initial choice for our experimental study of the complex logistic map (5). We study it in two cases for the values of the parameter $r$.

Case I. When $r$ is purely real, we compute the orbits of the map for fixed $\alpha$ and $\beta$ and go on varying $r$ until the iterate of the map remains bounded. These threshold values of $r$ are computed under 2000 iterations and shown in the Table 1.

Table 1. The optimum values of $|r|$ (when $r_{y}=0$ ) under 2000 iterations

\begin{tabular}{|c|c|c|c|c|c|c|}
\hline $\boldsymbol{\beta}$ & 1 & 0.9 & 0.7 & 0.5 & 0.3 & 0.1 \\
\hline 0 & $\underline{\mathbf{2 . 7 9 1 8}}$ & 3.0140 & 3.5438 & 4.3768 & 5.6343 & 11.9099 \\
\hline 0.1 & 3.1829 & $\mathbf{3 . 3 8 2 0}$ & $\underline{\mathbf{3 . 9 1 1 9}}$ & 5.9062 & 8.3954 & $\underline{\mathbf{1 8 . 7 9 0 1}}$ \\
\hline 0.3 & 3.2580 & 3.4635 & 4.0839 & 5.3423 & $\mathbf{7 . 0 2 2 8}$ & 11.0184 \\
\hline 0.5 & 3.3556 & 3.5767 & $\mathbf{3 . 9 8 0 4}$ & 4.5628 & 5.5696 & 8.4131 \\
\hline 0.7 & 3.1993 & 3.3453 & $\mathbf{3 . 5 6 8 7}$ & 4.0233 & $\mathbf{4 . 7 7 1 4}$ & 7.0142 \\
\hline 0.9 & $\underline{\mathbf{2 . 9 0 9 7}}$ & 3.0355 & 3.3343 & 3.8592 & $\underline{\mathbf{4 . 2 5 7 0}}$ & $\underline{\mathbf{6 . 2 2 0 9}}$ \\
\hline
\end{tabular}

In this case it is observed that for a fixed $\beta$ and varying $\alpha$ (from 1 towards zero), the optimum value of the control parameter $r$ increases surprisingly to a maximum of 18.7901. Further, on fixing $\alpha$ and varying $\beta$, the optimum value of $r$ shows an increment to some instant after which it starts decreasing (see Table 1). The corresponding fractal patterns for some random values of $r$ (shown bold) are drawn, although the same could be drawn for all the tabulated values of $r$. The time series analysis showing the behaviours of the map is also shown in Fig. 1 for some specific choices of the parameter $r$ (shown underlined). 




(a) $\alpha=1, \beta=0, r=2.7918$

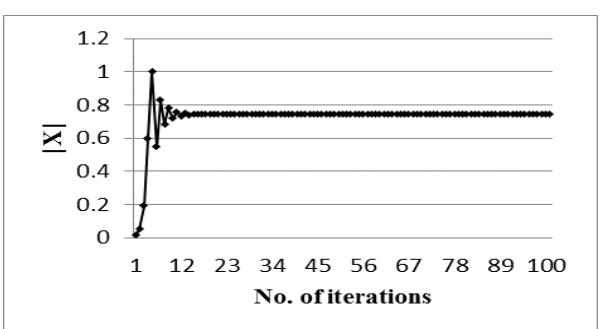

(c) $\alpha=0.7, \beta=0.1, r=3.9119$



(e) $\alpha=0.1, \beta=0.1, r=18.7901$



(b) $\alpha=1, \beta=0.9, r=2.9097$

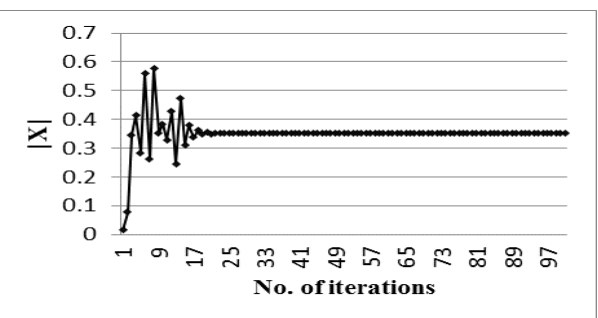

(d) $\alpha=0.3, \beta=0.9, r=4.2570$

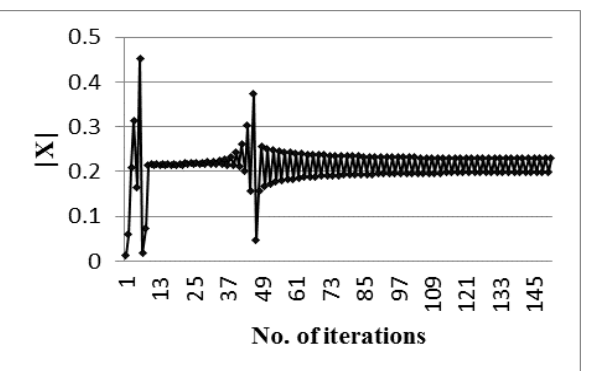

(f) $\alpha=0.1, \beta=0.9, r=6.2209$

Fig. 1. Time series at different values of $\alpha, \beta$ (when $r_{y}=0$ )

Case II. In this case, we obtain the optimal values of a purely imaginary $r$ for the same choices of the parameters $\alpha$ and $\beta$. We observe that for a fixed $\beta$ and varying $\alpha$ (from 1 towards zero), the optimum value of the magnitude of the control parameter $r$ increases to a maximum of 10.7525 for the same choice of $\alpha$ and $\beta$. Further, on fixing $\alpha$ and varying $\beta$, the optimum value of $r$ shows an increment to some instant after which it starts decreasing (Table 2).

Table 2. The optimum values of $|r|$ (when $r_{x}=0$ ) under 2000 iterations

\begin{tabular}{|c|c|c|c|c|c|c|}
\hline \multicolumn{1}{|c|}{$\boldsymbol{\alpha}$} & 1 & 0.9 & 0.7 & 0.5 & 0.3 & 0.1 \\
\hline 0 & $\underline{\mathbf{1 . 0 3 1 8}}$ & 1.1079 & 1.3651 & 1.8024 & 2.3884 & $\mathbf{4 . 3 9 9 7}$ \\
\hline 0.1 & 1.1049 & $\mathbf{1 . 2 3 5 8}$ & 1.5775 & 2.1436 & 3.4989 & $\underline{\mathbf{1 0 . 7 5 2 5}}$ \\
\hline 0.3 & 1.2592 & 1.4230 & $\underline{\mathbf{1 . 8 5 3 5}}$ & 2.5349 & $\underline{\mathbf{3 . 8 0 5 8}}$ & 7.6298 \\
\hline 0.5 & 1.2509 & 1.3979 & $\mathbf{1 . 7 6 3 6}$ & 2.2977 & 3.2410 & 6.0884 \\
\hline 0.7 & 1.1584 & 1.2859 & 1.5932 & 2.0378 & $\mathbf{2 . 8 1 8 7}$ & 5.1939 \\
\hline 0.9 & $\mathbf{1 . 0 5 2 0}$ & 1.1633 & 1.4346 & 1.8241 & 2.5078 & $\mathbf{4 . 5 9 3 7}$ \\
\hline
\end{tabular}


The corresponding fractal patterns for some random values of $|r|$ (shown bold) are drawn, although the same could be drawn for all the tabulated values of $|r|$. The time series analysis showing the behaviours of the map is also shown as Fig. 2 for some specific choices of the magnitude of the parameter $r$ (shown underlined).

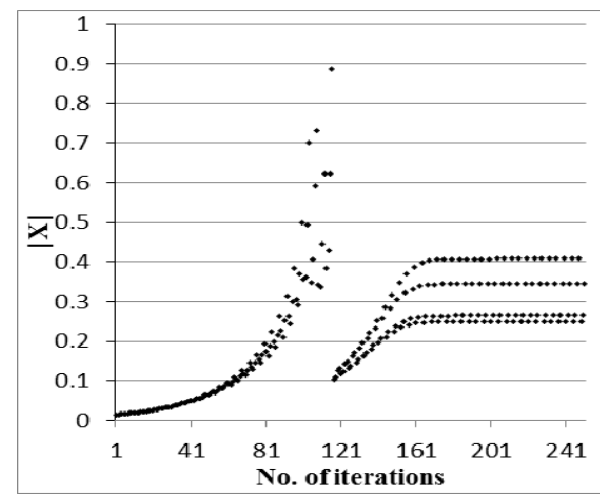

(a) $\alpha=1, \beta=0, r=1.0318 i$

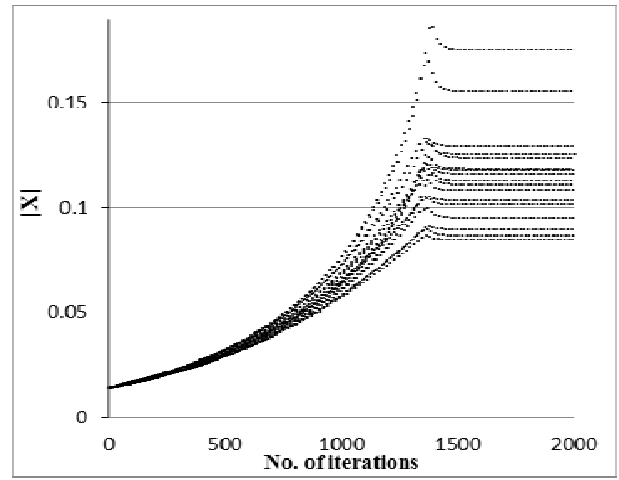

(i) for whole range of $|\mathrm{X}|$

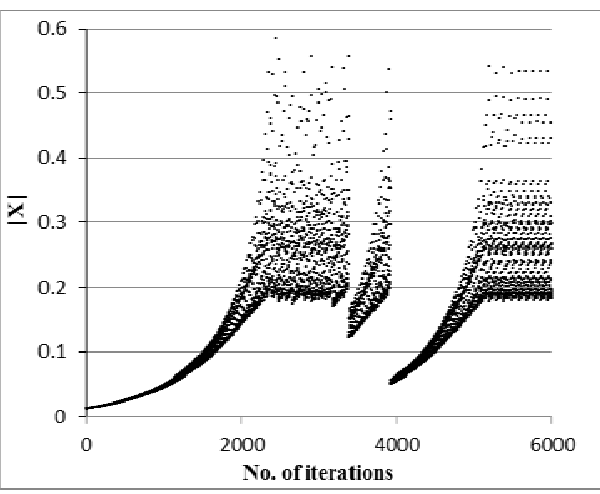

(b) $\alpha=0.7, \beta=0.3, r=1.8535 i$

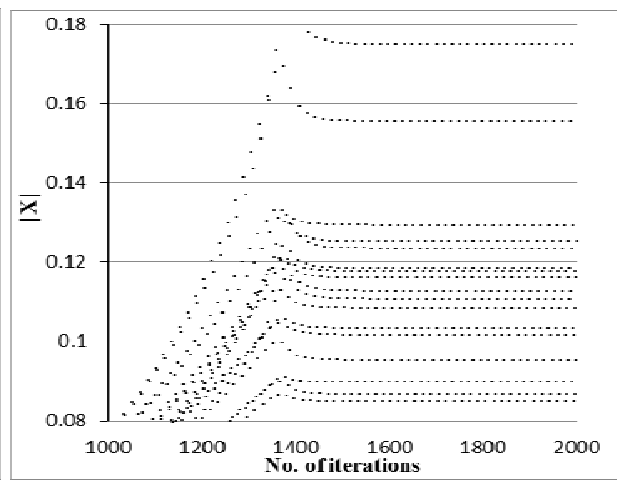

(ii) zoomed for $0.08 \leq|\mathrm{X}| \leq 0.18$

(c) $\alpha=0.3, \beta=0.3, r=3.8058 i$

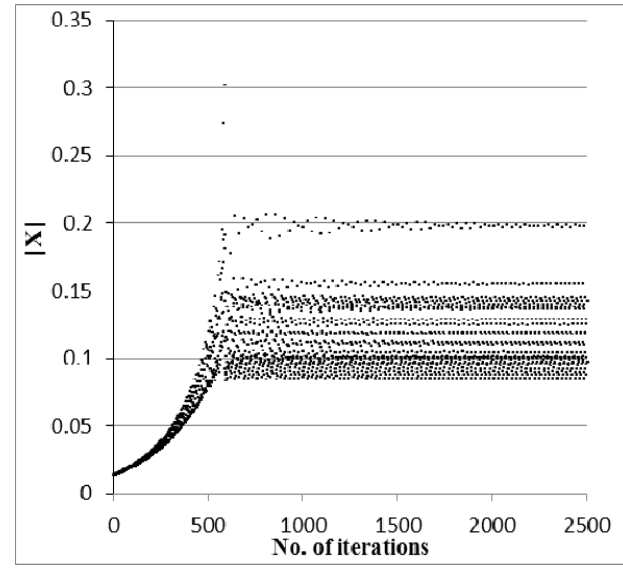

(i) for whole range of $|\mathrm{X}|$

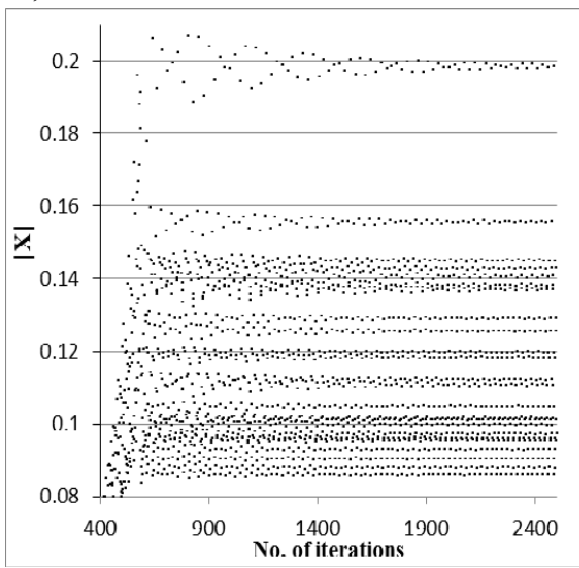

(ii) zoomed for $0.08 \leq|\mathrm{X}| \leq 0.21$

(d) $\alpha=0.1, \beta=0.1, r=10.7714 i$

Fig. 2. Time series at different values of $\alpha, \beta$ (when $r_{x}=0$ ) 
We also study the behaviour of the map for general complex $r$ by taking some selected values of $\alpha$ and $\beta$. The optimum value of $r$ is plotted for 72 directions with an angular increment of 5 degrees (Fig. 3).

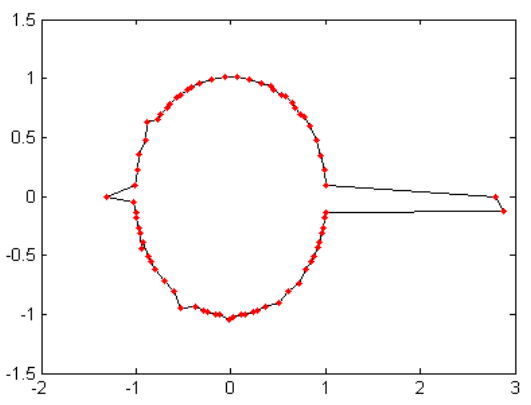

(a) $\alpha=1, \beta=0$

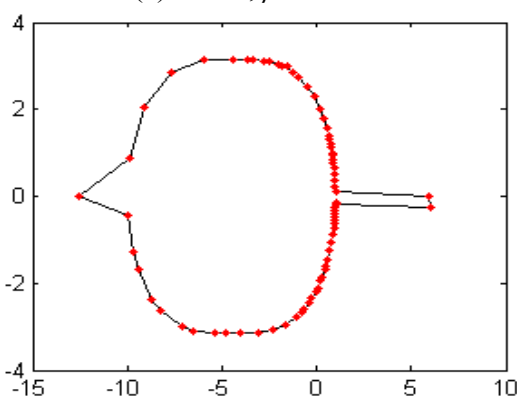

(c) $\alpha=0.5, \beta=0.1$



(e) $\alpha=0.1, \beta=0$

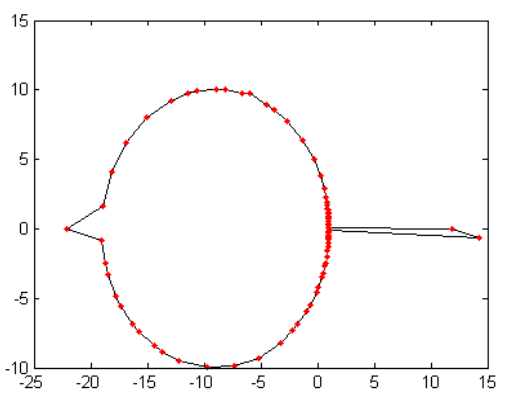

(b) $\alpha=1, \beta=0.9$

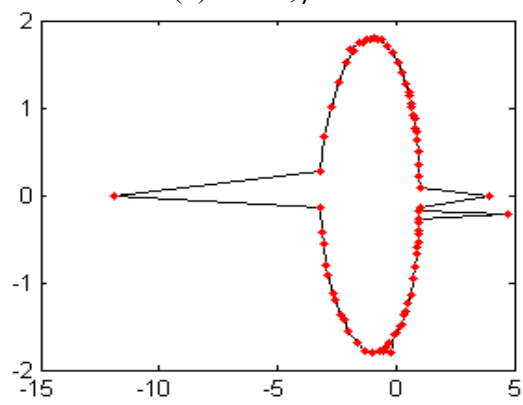

(d) $\alpha=0.7, \beta=0.1$

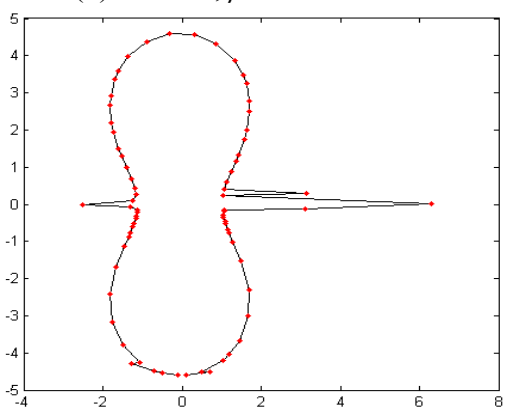

(f) $\alpha=0.1, \beta=0.9$

Fig. 3. Plots of the optimum values of $r$ (with increment 5 degrees) under 2000 iterations

Now we study the fractal analysis of the map defined in (5) and generate the fractal patterns for (6) by obtaining the value of the parameter $c$ from the tabulated value of $r$ (Tables 1-2) using $c=r(2-r) / 4$. Devaney [7] defined the escape criteria for Picard iterate of the complex quadratic map and observed that the orbit escapes when $\left|z_{n}\right| \geq|c|>2$. So, when $\left|z_{n}\right| \geq|c|>2$ for some $n$, then $\left|z_{n}\right| \rightarrow \infty$ as $n \rightarrow \infty$. Attractive fractal patterns are obtained by them on the basis of this escape algorithm. We extend it for the Ishikawa iterates of the complex quadratic maps and found that the orbit escapes when $\left|z_{n}\right|>\max \{c \mid, 2 / \alpha, 2 / \beta\}$ where $0<\alpha \leq 1,0 \leq \beta \leq 1$. Therefore, if we construct a sequence $\left\{z_{n}\right\}$ using Ishikawa iteration with $\left|z_{n}\right|>\max \{c \mid, 2 / \alpha, 2 / \beta\}$ for some $n$, then $\left|z_{n}\right| \rightarrow \infty$ as $n \rightarrow \infty$. We follow the colour 
schemes of Pietgen and Saupe [22] along with the above defined escape criterion for our study of the complex logistic map. The colouring scheme of the graphics presented in the figures depends upon the rate of escape to infinity. A point $z_{0}$ is coloured black if the orbit of $z_{0}$ not escaped within the first 100 iterates, red is used to denote points which escape to infinity fastest. Shades of orange, yellow and green are used to colour points which escaped less quickly and shades of blue and violet represent the points which escaped, but only after a significant number of iterations. This colouring scheme is well depicted in the graphical patterns given in Figs 4 and 5.

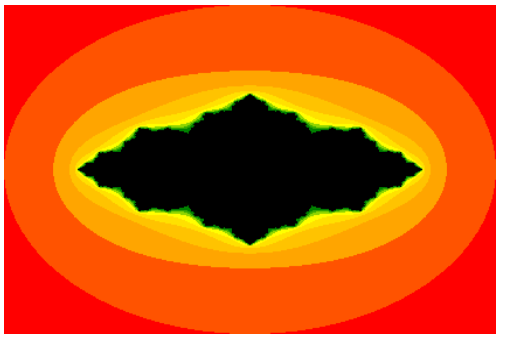

(a) $\alpha=1, \beta=0, r=2.7918$

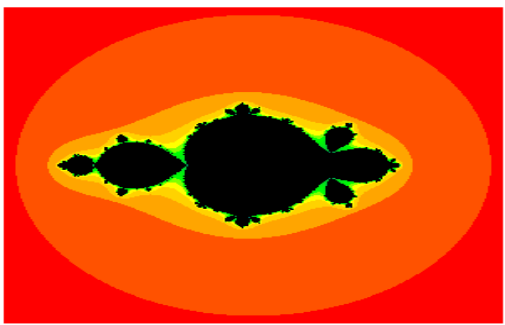

(c) $\alpha=0.7, \beta=0.7, r=3.5687$

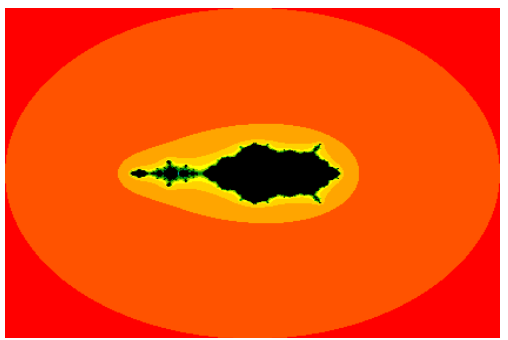

(e) $\alpha=0.3, \beta=0.7, r=4.7714$

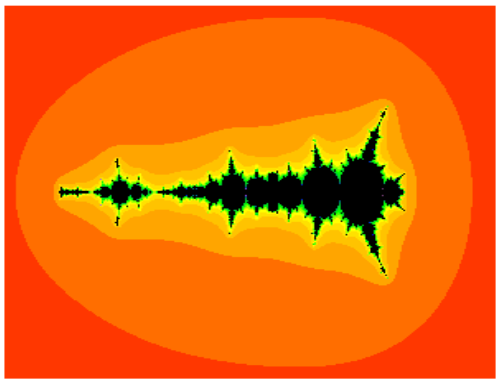

(g) $\alpha=0.1, \beta=0.9, r=6.2209$

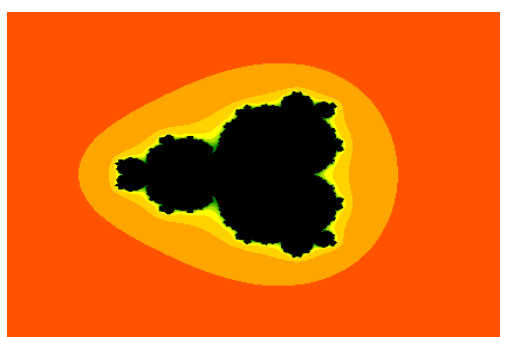

(b) $\alpha=0.9, \beta=0.1, r=3.3820$

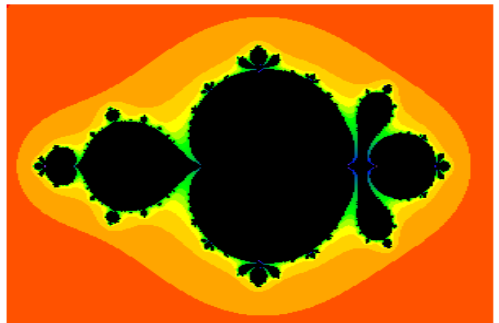

(d) $\alpha=0.7, \beta=0.5, r=3.9804$

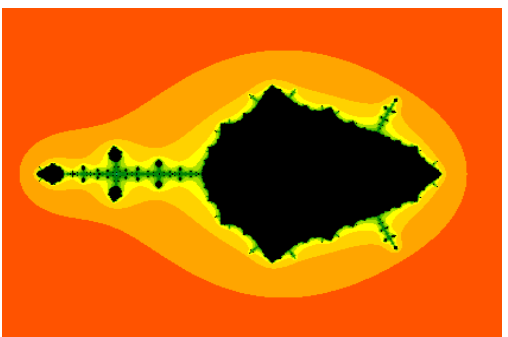

(f) $\alpha=0.3, \beta=0.3, r=7.0228$

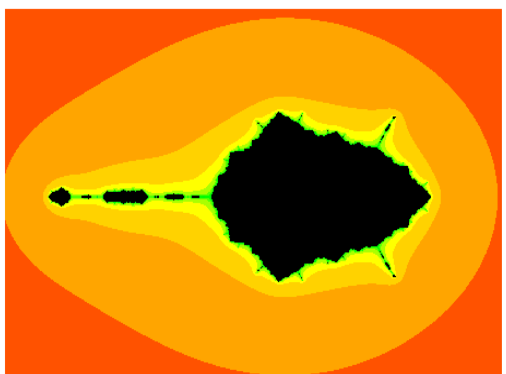

(h) $\alpha=0.1, \beta=0.1, r=18.7901$

Fig. 4. Julia sets for real $r$ 


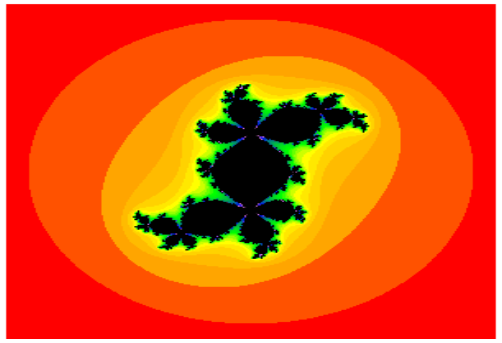

(a) $\alpha=1, \beta=0, r=1.0318 i$

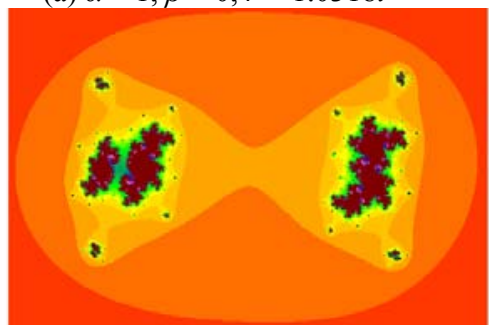

(c) $\alpha=0.9, \beta=0.1, r=1.2358 i$

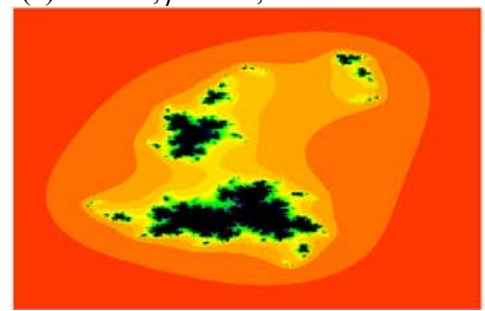

(e) $\alpha=0.3, \beta=0.7, r=2.8187 i$

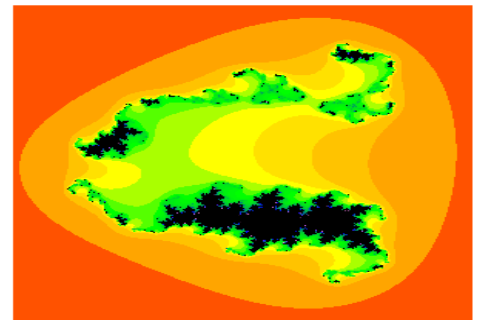

(g) $\alpha=0.1, \beta=0.1, r=10.7525 i$

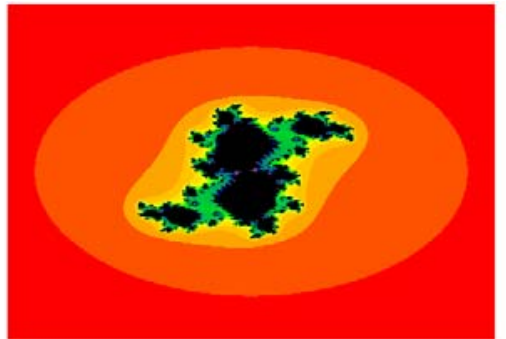

(b) $\alpha=1, \beta=0.9, r=1.0520 i$

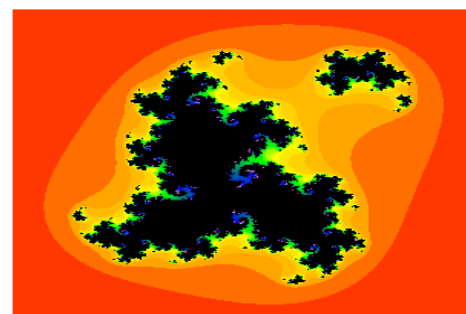

(d) $\alpha=0.7, \beta=0.5, r=1.7636 i$

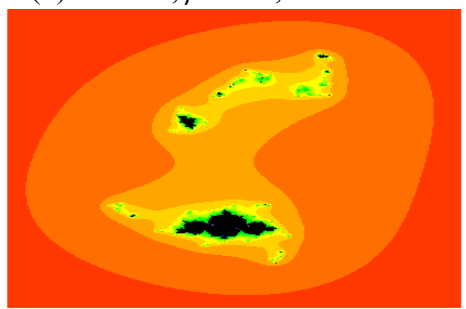

(f) $\alpha=0.1, \beta=0.9, r=4.5937 i$

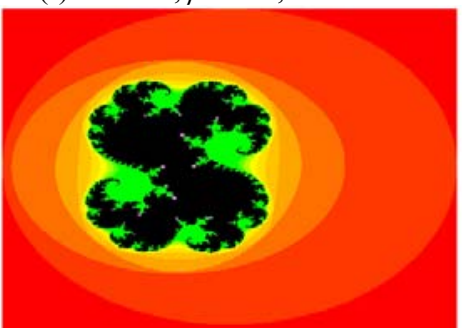

(h) $\alpha=0.1, \beta=0, r=4.3997 i$

Fig. 5. Julia sets for purely complex $r$

\section{Conclusion}

We observe that for a fixed $\beta$ and varying $\alpha$ (from 1 towards zero), the optimum value of the magnitude of the control parameter $r$ (in purely real case) increases surprisingly to a maximum of 18.7901 whereas it increases to a maximum of 10.7525 in case of purely imaginary $r$ for the same choice of $\alpha$ and $\beta$. Further, on fixing $\alpha$ and varying $\beta$ the optimum value of $r$ shows an increment to some instant after which it starts decreasing (see, Tables 1 and 2) for both the cases. The time series analysis of the complex logistic map confirms the bounded behaviour of the logistic map even for the higher values of $|r|$ for specific choices of the parameters $\alpha$ and $\beta$. 
Acknowledgements: The authors would like to thank the learned referees for their valuable comments and suggestions for the improvement of this manuscript.

\section{References}

1. B a rnsley, M. F. Fractals Everywhere. Second Ed. Revised with the Assistance of and a Foreword by Hawley Rising, III. Boston MA, Academic Press Professional, 1993.

2. B a r n s le y, M. F. Superfractals. Cambridge, Cambridge University Press, 2006.

3. A. Bunde, S. Havlin, Eds. Fractals in Science. Springer-Verlag, 1994.

4. C a m a c h o, E. F., C. B or d o n s. Model Predictive Control. Berlin, Springer, 1999.

5. C r o w n o ve r, R. M. Introduction to Fractals and Chaos. Jones \& Barlett Publishers, 1995.

6. D e t t $\mathrm{m}$ e r, R. Chaos and Engineering. - IEE Review, September 1993, 199-203.

7. Devaney, R. L. A First Course in Chaotic Dynamical Systems: Theory and Experiment. Addison-Wesley, 1992.

8. Fe ig e $\mathrm{nb}$ a u m, M. Quantitative Universality for a Class of Non-Linear Transformations. J. Statistical Physics, Vol. 19, 1978, 25-52.

9. H o $1 \mathrm{~m}$ g r e n, R. A. A First Course in Discrete Dynamical Systems. Springer-Verlag, 1994.

10. Is hik aw a, S. Fixed Points by a New Iteration Method. - Proc. Amer. Math. Soc., Vol. 44, 1974, No 1, 147-150.

11. J u 1 i e n, C. S. Chaos and Time-Series Analysis. Oxford University Press, 2003.

12. K e 11 e r, K. Invariant Factors, Julia Equivalences, and the (Abstract) Mandelbrot Set. - Berlin Heidelberg New York, Springer-Verlag, 2000.

13. Kint, J., D. Constales, A. Vanderbauwhede. Pierre-Francois Verhulst's Final Triumph. - In: M. Ausloos, M. Dirickx Eds. The Logistic Map and the Route to Chaos: From the Beginnings to Modern Applications. Springer-Verlag, 2006.

14. M a n n, W. R. Mean Value Methods in Iteration. - Proc. Amer. Math. Soc., Vol. 4, 1953, No 3, 506-510.

15. M a y, R. M. Simple Mathematical Models with Very Complicated Dynamics. - Nature, Vol. 261 1976, No 459, 459-475.

16. M a y, R. M., G. F. O s t e r. Bifurcations and Dynamic Complexity in Simple Biological Models. - The American Naturalist, Vol. 110, 1976, No 974, 573-599.

17. Moon ey, A., J. G. Ke ating, D. M. He ffernan. A Detailed Study of the Generation of Optically Detectable Watermarks Using the Logistic Map. - Chaos, Solitons and Fractals, Vol. 30, 2006, No 5, 1088-1097.

18. M or a n, P. A. P. Some Remarks on Animal Population Dynamics. - Biometrics, Vol. 6, 1950, No 3, 250-258.

19. P a r e e k, N. K., V. P a ti d a r, K. K. S u d. Image Encryption Using Chaotic Logistic Map. Image and Vision Computing, Vol. 24, 2006, No 9, 926-934.

20. P a s t i j n, H. Chaotic Growth with the Logistic Model of P.-F. Verhulst. - In: M. Ausloos, M. Dirickx, Eds. The Logistic Map and the Route To Chaos: From the Beginnings to Modern Applications. - Springer-Verlag, 2006.

21. Peitgen, H., H. Jurgens, D. S a upe. Chaos and Fractals: New Frontiers of Science. Springer-Verlag, 2004.

22. H. Peitgen, D. Saupe, Eds. The Science of Fractal Images. Springer-Verlag, 1988.

23. Pr a s a d, B., K. K a t i y a r. A Comparative Study of Logistic Map Through Function Iteration. In: Proc. Int. Con. Emerging Trends in Engineering and Technology. ISBN: 978-93-8069722-2, Kurukshetra, India, 2010, 357-359.

24. P r a s a d, B., K. K a t i y a r. Fractals via Ishikawa Iteration. - CCIS, Springer, Berlin, Heidelberg, Vol. 140, 2011, No 2, 197-203.

25. Pras a d, B., K. K a ti y ar. A Stability Analysis of Logistic Model. - International Journal of Nonlinear Science, Vol. 17, 2014, No 1, 71-79.

26. R a n i, M., R. A g a r w a l. A New Experimental Approach to Study the Stability of Logistic Map. - Chaos, Solitons and Fractals, Vol. 41, 2009, No 4, 2062-2066.

27. R a n i, M., R. A g a rw a l. Generation of Fractals from Complex Logistic Map. - Chaos, Solitons and Fractals, Vol. 42, 2009, No 1, 447-452.

28. S a l a ri e h, H., M. Shahrokhi. Indirect Adaptive Control of Discrete Chaotic Systems. Chaos, Solitons and Fractals, Vol. 34, 2007, No 4, 1188-1201. 